\title{
Coding practice of the Journal Article Tag Suite extensible markup language
}

\author{
Sun Huh \\ Department of Parasitology and Institute of Medical Education, College of Medicine, Hallym University, Chuncheon, Korea
}

\section{Abstract}

In general, the Journal Article Tag Suite (JATS) extensible markup language (XML) coding is processed automatically by an XML filtering program. In this article, the basic tagging in JATS is explained in terms of coding practice. A text editor that supports UTF-8 encoding is necessary to input JATS XML data that works in every language. Any character representable in Unicode can be used in JATS XML, and commonly available web browsers can be used to view JATS XML files. JATS XML files can refer to document type definitions, extensible stylesheet language files, and cascading style sheets, but they must specify the locations of those files. Tools for validating JATS XML files are available via the web sites of PubMed Central and ScienceCentral. Once these files are uploaded to a web server, they can be accessed from all over the world by anyone with a browser. Encoding an example article in JATS XML may help editors in deciding on the adoption of JATS XML.

Keywords

Cascading style sheets; Document type definition; Extensible stylesheet language; Journal Article Tag Suite; Journal publishing

Received: June 5, 2014

Accepted: July 29, 2014

Correspondence to Sun Huh shuh@hallym.ac.kr

ORCID

Sun Huh

http://orcid.org/0000-0002-8559-8640

This article was presented at the workshop entitled 'Application of advanced

information technology to scholarly journal publishing' during the 12th EASE General Assembly and Conference, Split, Croatia, July 12-13, 2014.

\section{Introduction}

Anyone who can understand English and has a modest knowledge of journal editing and publishing can produce Journal Article Tag Suite (JATS) extensible markup language (XML) files. Manually coding such documents according to a document type definition (DTD) requires 12 hours for a single article, even if any existing extensible hypertext markup language (XHTML), mathematical markup language (MathML), and chemical markup language (ChemML) portions are treated as figure formats. Therefore, most printing companies employ a special XML conversion program. In general, the preliminary XML file for a single article is generated within 15 minutes. Then, the validation check and trimming of the file is performed. XML coding of articles requires some knowledge of bibliographic formatting conventions in order to differentiate the bibliographic characteristics of data in the articles. For example, in the reference section, journals, books, web sites, or $\mathrm{PhD}$ theses may be cited. Typically, these distinct reference 
types should be formatted differently. The objective of this article is to explain how editors can code journal articles according to JATS 1.0 XML specification and to observe the results through a web browser. In particular, at least $20 \mathrm{XML}$ tags are considered and the role of DTDs, extensible stylesheet language transformations (XSLT), and cascading style sheets (CSS) are explained. It is expected that after gaining some experience with the article coding process, editors will have an incentive to adopt JATS XML.

\section{Programs and Sample File}

An editor program that supports Unicode and a web browser are necessary for coding JATS XML file. JATS XML sample files are provided as Supplement 1. Further, the DTD, XSLT, and CSS files are available as well: a journalpublishing1.dtd file was available from https://github.com/PeerJ/jats-conversion/ blob/master/schema/jats/publishing/1.0/JATS-journalpublishing1.dtd; a jats-html.xsl file was available from: https:// github.com/ncbi/JATSPreviewStylesheets/blob/master/xslt/ main/jats-html.xsl; and a jats-preview.css file was available from: https:/github.com/wendellpiez/oXygenJATSframework/blob/master/jats-preview-xslt/jats-preview.css.

\section{Coding an XML File and Browsing Using DTD, XSLT, and CSS}

First, open the sample coding article within your text editor. Save the file (sample.xml) to a specific directory, ensuring that UTF-8 encoding is maintained. Open the file in a web browser and observe how it appears. It is presented as Fig. 1. Next, copy the DTD file (journalpublishing1.dtd) into the same directory where the sample file is located. Re-open the sample file in a browser and observe how its appearance has changed.
To the same directory, add the XSLT file (jats-html.xsl) and then the CSS file (jats-preview.css). Each time re-open sample. $\mathrm{xml}$ in a browser and observe any changes (Figs. 2, 3). With each additional file, you should note any improvements in the layout and appearance of the document, and these changes should suggest the function of each file added.

Since a DTD provides the attributes and elements only, the format of the sample file as viewed in the browser does not change (Fig. 1). XSLT files, in contrast, typically define how an XML document is to be rendered or transformed into hypertext markup language (HTML) for example (Fig. 2). Finally, CSS files describe the look and formatting of documents written in mark-up languages such as HTML (Fig. 3).

\section{How to Declare an XML Document?}

An XML document begins as follows:

$<$ ?xml version = "1.0" encoding = "UTF-8"? >---(1)

$<$ ?xml-stylesheet type $=$ "text/xsl" href = "jats-html.xsl"? $>$ ---(2)

$<$ !DOCTYPE article PUBLIC “-//NLM//DTD JATS (Z39. 96) Journal Publishing DTD v1.0 20120330//EN” “http:// jats.nlm.nih.gov/publishing/1.0/JATS-journalpublishing1.dtd">---(3)

(1) < ?xml version $=$ " 1.0 " encoding $=$ "UTF-8"? > means that the $\mathrm{xml}$ version is 1.0 and encoding is according to UTF-8. It is the most common declaration for XML documents.

(2) The $<$ ?xml-stylesheet type = "text/xsl" href = "jats-html. xsl"? > tag determines the stylesheet. Layout is assigned in jats-html.xsl.

(3) The declaration on line three beginning with ' $<$ !DOCTYPE article' indicates the online location of the DTD
KCSE Science Editing 2288-7474 2288-9063 Korean Council of Science Editors http://dx.doi.org/10.6087/kcse.2014.1.15 sciedit-1-15 Educational Material Coding practice of Journal Article Tag Set eXtensible Markup Language HuhSun $1 * 1$ Department of Parasiotology and Institute of Medical Education, College of Medicine, Hallym University, Chuncheon, Korea * shuh@hallym.ac.kr 2014200820141150406201426 062014 (C) 2014, Korean Council of Science Editors 2014 This is an open-access article distributed under the terms of the Creative Commons Attribution License, which permits unrestricted use, distribution, and reproduction in any medium, provided the original work is properly cited. Journal Article Tag Set eXtensible Markup Language (JATS XML) coding is usually processed automatically by XML filtering program and the accuracy of tagging is checked by validation process.... Journal Article Tag Suite journal publishing Introduction Production of Journal Article Tag Set eXtensible Markup Language (JATS $\mathrm{XML}$ ) files can be done by anyone who can understand English and have minimum knowledge on journal editing and publishing.... Programs and sample files Unicode supporting text editor. Edit plus (R) and web browser, Firefox are necessary. Conclusion JATS XML coding can be done according to the JATS DTD which provide the

Fig. 1. Screenshot of sample.xml file seen with browser when there was no other file or journalpublishing1.dtd file at the same directory with sample.xml. 


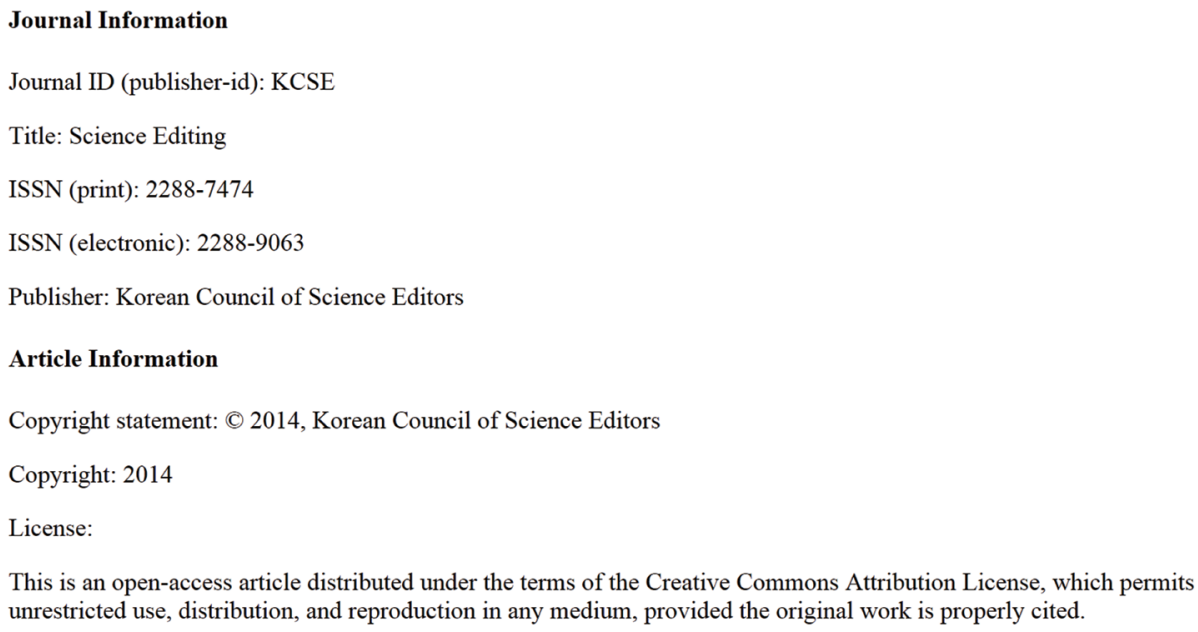

Fig. 2. Screenshot of sample.xml file seen when there were journalpublishing1.dtd and jats-html.xsl files at the same directory with sample.xml.

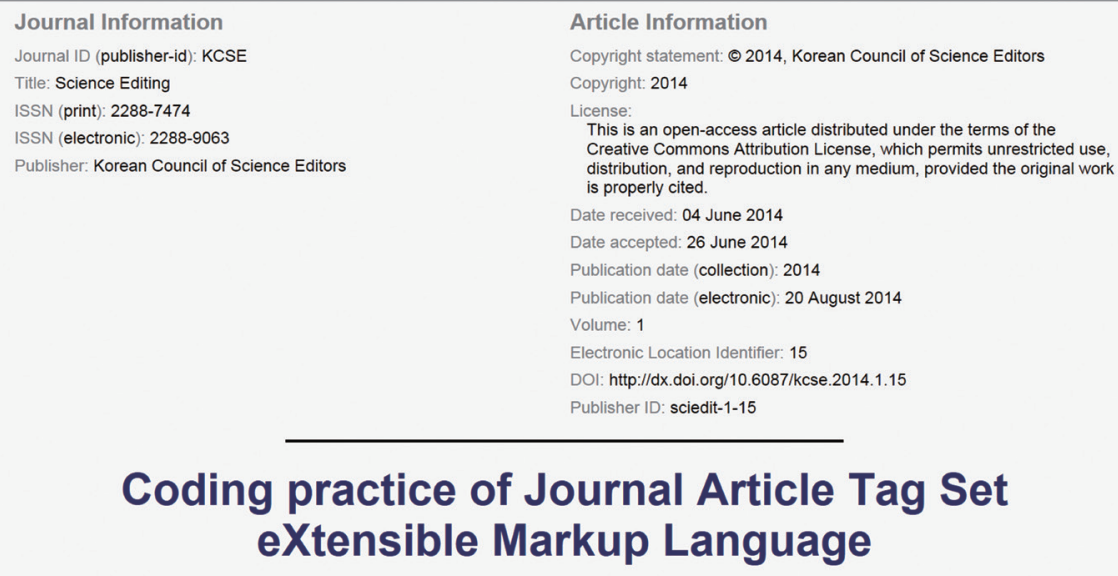

\section{Coding practice of Journal Article Tag Set eXtensible Markup Language}

Sun Huh['][1]

[1] Department of Parasiotology and Institute of Medical Education,

Fig. 3. Screenshot of sample.xml file seen when there were journalpublishing1.dtd, jats-html.xsl, and jats-preview.css files at the same directory with sample.xml.

file. If the DTD file is instead located on a local file system, the declaration would appear as <!DOCTYPE article PUBLIC “-//NLM//DTD JATS (Z39.96) Journal Publishing DTD v1.0 "JATS-journalpublishing1.dtd” > The DTD determines the elements and attributes that are permissible within any XML document that refers to it. Previously, different DTDs were used by each publisher; however, the JATS XML DTD is now conventionally used.

\section{Article Declaration}

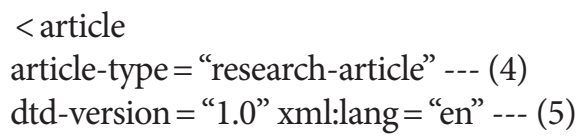

xmlns:mml = http://www.w3.org/1998/Math/MathML $---(6)$ xmlns:xlink=http://www.w3.org/1999/xlink --- (7) xmlns:xsi = http://www.w3.org/2001/XMLSchema-instance $---(8)$

$>$

Above is an example of an article declaration.

(4) article-type = "research-article" specifies that the publication type is "research article." A variety of types are available, such as "editorial," "letter," and "case report."

(5) dtd-version = "1.0" xml:lang = "en" declares the language in which the article is written. If there is no language declaration, the default value is "en" (English). 
(6-8) The last three lines in the article declaration above indicate that this article follows W3C MathML, XLINK, and XML Schema-instance.

\section{Tag Construction}

Articles are comprised of three sections classified as "front matter," "body," and "back matter." Each of these sections is enclosed within the corresponding tag pair as follows:

$$
\begin{aligned}
& <\text { front }>---(9) \\
& \ldots . \\
& </ \text { front }> \\
& <\text { body }>---(10) \\
& \ldots \\
& </ \text { body }> \\
& <\text { back }>--(11) \\
& \ldots \\
& </ \text { back }>
\end{aligned}
$$

(9) "Front matter" consists of citation details, an abstract, keywords, and masthead.

(10) The "body" section includes the article's primary content from the introduction to conclusion.

(11) "Back matter" comprises conflict of interest, acknowledgments, footnotes, references, appendices, and/or supplements.

\section{Special Characters}

Special characters such as $*,<$, and $>$ must be specified using the corresponding Unicode character entity reference, for example:

$$
\begin{aligned}
& * \quad \& \# \mathrm{x} 002 \mathrm{~A} ; \\
& <\quad \& \# \mathrm{x} 003 \mathrm{C} ; \\
& >\quad \text { \&\#x003e; }
\end{aligned}
$$

All Unicode character entity references can be specified with a four-digit hexadecimal code, prepended with \&\# $\mathrm{x}$ and appended with a semicolon. For example, the code for the \% character is 0025 ; therefore, the complete character entity reference is \&\#x0025;. A full list of codes for all special characters is available at http://www.unicode.org/charts/.

\section{Use of Local Language}

To make a full-text JATS XML file for articles in Croatian, it is necessary to add a language tag such as $<\mathrm{xml} l \mathrm{lang}=$ "hr" $>$ in the article declaration. The language element is specified as an attribute with a two-letter alphabetic code in accordance with the IETF RFC 5646 (http://tools.ietf.org/html/rfc5646) recommended by the Internet Engineering Task Force in Sep- tember 2009. For example, "fr" (French), "en" (English), "de" (German), "se" (Swedish), "hr" (Croatian), "es" (Spanish), and "ko" (Korean) were used [1].

\section{Tagging Practice}

After adding the journal article's content to the appropriate sections of the sample file, you can then check how it appears in a web browser.

\section{Validation of JATS XML}

Once the JATS XML file has been produced, it can be validated with any variety of tools available online, such as http:// www.ncbi.nlm.nih.gov/pmc/tools/xmlchecker/ or http:// www.e-sciencecentral.org/tools/stylechecker/. Any indicated errors should be fixed in accordance with the JATS DTD.

\section{Why Is It Necessary to Establish a JATS XML Producing Company That Deals with Each Language?}

There is still small portion of full-text JATS XML-based society-directed journals in the web. A number of scientific journal publishing societies in Korea have begun to produce fulltext JATS XML files and deposit them to ScienceCentral, since at least three Korean firms can generate perfect JATS XML files with table XHTML, ChemML, and MathML [2]. It is the base of producing JATS XML files both in English and in Korean. There are other excellent global companies that can produce JATS XML files; however, they usually process articles in English. Therefore, JATS XML-producing company of which specialists fluent in each language is needed in order to make articles accessible internationally via the Web and to deposit them to ScienceCentral for wider exposure.

\section{Conclusion}

JATS XML coding can be performed according to the JATS DTD, which provides the specification for elements and attributes. XSLT provides the stylesheet for XML; whereas CSS provides the stylesheet for HTML. With DTD, XSLT, and CSS, the JATS XML file can be viewed in a user-friendly fashion via a web browser. Journals in local languages can be specified in JATS XML files with the appropriate code in the language attribute. Such journals will then be accessible to all readers in the world with a variety of formats including PubReader and ePub. 


\section{Conflict of Interest}

No potential conflict of interest relevant to this article was reported.

\section{Acknowledgments}

This work is supported by a research grant of the National Research Foundation of Korea (policy research-2013-003-academic infrastructure promotion) and the Korean Federation of Science and Technology Societies (KOFST-2013), government of the Republic of Korea.

\section{References}

1. Huh S, Choi TJ, Kim SH. Using Journal Article Tag Suite extensible markup language for scholarly journal articles written in Korean. Sci Ed 2014;1:19-23. http://dx.doi.org/ 10.6087/kcse.2014.1.19

2. Huh S. Application of new information technologies to scholarly journals: ORCID, CrossMark, and FundRef. J Korean Med Assoc 2014;57:455-62. http://dx.doi.org/10. 5124/jkma.2014.57.5.455 
Supplement 1. Sample file for exercise of Journal Article Tag Suite

$<$ ?xml version="1.0" encoding = "UTF-8"? >

$<$ ?xml-stylesheet type = "text/xsl" href="jats-html.xsl"? >

$<$ !DOCTYPE article PUBLIC “-//NLM//DTD JATS (Z39.96) Journal Publishing DTD v1.0 20120330//EN" "journalpublishing1.dtd" >

$<$ article

article-type = "research-article"

dtd-version="1.0" xml:lang ="en"

xmlns:mml = "http://www.w3.org/1998/Math/MathML"

xmlns:xlink= "http://www.w3.org/1999/xlink"

xmlns:xsi = "http://www.w3.org/2001/XMLSchema-instance"

$<$ front $>$

$<$ journal-meta $>$

$<$ journal-id journal-id-type $=$ "publisher-id" $>$ KCSE $</$ journal-id $>$

$<$ journal-title-group $><$ journal-title $>$ Science Editing $</$ journal-title $><$ /journal-title-group $>$

$<$ issn pub-type $=$ "ppub" $>2288-7474<$ /issn $>$

$<$ issn pub-type $=$ "epub" $>2288-9063</$ issn $>$

$<$ publisher $>$

$<$ publisher-name $>$ Korean Council of Science Editors $</$ publisher-name $></$ publisher $>$

$</$ journal-meta $>$

$<$ article-meta $>$

$<$ article-idpub-id-type $=$ "doi" $>$ 10.6087/kcse.2014.1.15</article-id $>$

$<$ article-id pub-id-type $=$ "publisher-id" $>$ sciedit-1-15</article-id $>$

$<$ article-categories >

$<$ subj-group $>$

$<$ subject $>$ Educational Material $</$ subject $>$

$</$ subj-group $>$

$</$ article-categories $>$

$<$ title-group $>$

$<$ article-title > Coding practice of Journal Article Tag Set eXtensible Markup Language

$<$ /article-title $>$

$</$ title-group $>$

$<$ contrib-group $>$

$<$ contrib contrib-type $=$ "author" $>$

$<$ name $><$ surname $>$ Huh $<$ /surname $><$ given-names $>$ Sun $</$ given-names $><$ /name $>$

$<$ xref rid = "af1-sciedit-1-15" ref-type $=$ "aff" $><$ sup $>1</$ sup $></$ xref $>$

$<$ xref rid ="c1-sciedit-1-15" ref-type $=$ "corresp" $><$ sup $>\& \# x 0002 A ;</$ sup $></$ xref $>$

$</$ contrib $>$

$<$ aff id="af1-sciedit-1-15" >

$<$ label $>1</$ label $>$ Department of Parasitology and Institute of Medical Education, College of Medicine, Hallym University, Chuncheon,

$<$ country $>$ Korea $</$ country $>$

$</$ aff $>$

$</$ contrib-group $>$

$<$ author-notes >

$<$ corresp id ="c1-sciedit-1-15" >

$<$ label $>\& \# x 002 \mathrm{~A} ;</$ label $><$ email $>$ shuh@hallym.ac.kr</email $></$ corresp $>$

$<$ /author-notes $>$

$<$ pub-date pub-type $=$ "collection" $>$

$<$ year $>2014<$ /year $><$ /pub-date $>$

$<$ pub-date pub-type $=$ "epub" $>$

$<$ day $>20<$ day $>$

$<$ month $>08<$ /month $>$

$<$ year $>2014<$ /year $><$ /pub-date $>$

$<$ volume $>1</$ volume $>$

$<$ elocation-id $>15<$ /elocation-id >

$<$ history $>$

$<$ date date-type = "received" $>$ 
Supplement 1. Continued

$<$ day $>04</$ day $>$

$<$ month $>06<$ /month $>$

$<$ year $>2014</$ year $></$ date $>$

$<$ date date-type $=$ "accepted" $>$

$<$ day $>26<$ day $>$

$<$ month $>06<$ /month $>$

$<$ year $>2014</$ year $><$ /date $><$ /history $>$

$<$ permissions $>$

$<$ copyright-statement > \&\#x00A9; 2014, Korean Council of Science Editors</copyright-statement $>$

$<$ copyright-year $>2014<$ /copyright-year $>$

$<$ license $>$

$<$ license-p $>$ This is an open-access article distributed under the terms of the Creative Commons Attribution License, which permits unrestricted use, distribution, and reproduction in any medium, provided the original work is properly cited. $</$ license- $p></$ license $></$ permissions $>$

$<$ abstract>

$<\mathrm{p}>$

Journal Article Tag Set eXtensible Markup Language (JATS XML) coding is usually processed automatically by XML filtering program and the accuracy of tagging is checked by validation process.....

$</ \mathrm{p}>$

$<$ /abstract $>$

$<$ kwd-group $>$

$<$ kwd $>$ Journal Article Tag Suite $</$ kwd $>$

$<$ kwd $>$ journal publishing $</$ kwd $>$

$</$ kwd-group $>$

$</$ article-meta $>$

$</$ front $>$

$<$ body $>$

$<$ sec sec-type = "intro" $>$

$<$ title $>$ Introduction $</$ title $>$

$<\mathrm{p}>$

Production of Journal Article Tag Set eXtensible Markup Language (JATS XML) files can be done by anyone who can understand English and have minimum knowledge on journal editing and publishing....

$</ p>$

$</ \sec >$

$<$ sec sec-type $=$ "methods" $>$

$<$ title $>$ Programs and sample files $</$ title $>$

$<p>$ Unicode supporting text editor and web browser are necessary.

$</ p>$

$</ \sec >$

$<$ sec sec-type $=$ "conclusion" $>$

$<$ title $>$ Conclusion $</$ title $>$

$<\mathrm{p}>$

JATSXML coding can be done according to the JATSDTD which provide the information of elements and attributes.

$</ \mathrm{p}>$

$</$ sec $>$

$</$ body $>$

$<$ back $>$

$<$ fn-group $><$ fn fn-type $=$ "conflict" $>$

$\langle\mathrm{p}\rangle$ No potential conflict of interest relevant to this article was reported $</ \mathrm{p}\rangle</$ fn $\rangle</$ fn-group $>$

$<$ ack $>$

$<p>$ This work is supported by the research grant of the National Research Foundation of Korea (policy research-2013-003-academic infrastructure promotion) and the Korean Federation of Science and Technology Societies (KOFST-2013), Government of the Republic of Korea. $</ p>$

$</$ ack $>$

$<$ ref-list $>$

$<$ ref id="b1-sciedit-1-15" $>$

$<$ label $>1<$ /label $>$ 


\section{Supplement 1. Continued}

$<$ element-citation publication-type $=$ "journal" $>$ $<$ person-group person-group-type = "author" $>$

$<$ name $>$

$<$ surname $>$ Huh $<$ /surname $>$

$<$ given-names $>$ Sun $</$ given-names $>$

$<$ name $>$

$<$ name $>$

$<$ surname $>$ Choi $<$ /surname $>$

$<$ given-names $>$ Tae-Jin </given-names $>$

$<$ /name $>$

$<$ name $>$

$<$ surname $>$ Kim $<$ /surname $>$

$<$ given-names $>$ So-Hyung $<$ /given-names $>$

$</$ name $>$

$</$ person-group $>$

$<$ article-title $>$

Using Journal Article Tag Suite extensible markup language for scholarly journal articles written in Korean $</$ article-title $>$

$<$ source $>$ Sci Ed $</$ source $>$

$<$ year iso-8601-date $=$ "2014" $>2014</$ year $>$

$<$ volume $>1<$ /volume $>$

$<$ fpage $>19</$ fpage $>$

$<$ lpage $>23</$ lpage $>$

$</$ element-citation $>$

$</$ ref $>$

$</$ ref-list $>$

$</$ back $>$

$</$ article $>$ 